\title{
Early diagnosis Gorlin-Goltz syndrome: A Rare Case Report
}

\author{
Muhammed A Hussein ${ }^{1}$, Shakhawan M. Ali ${ }^{2 *}$, Delan J Anwer ${ }^{3}$ and Payman Kh Mahmud ${ }^{3}$ \\ ${ }^{1}$ Lecturer at Maxillofacial surgery department, Rizgary teaching hospital, Erbil, Kurdistan region, Iraq \\ ${ }^{2}$ Maxillofacial Department, KBMS trainee, Sulaimany teaching hospital, Sulaimany, Kurdistan region, Iraq \\ ${ }^{3}$ Oral medicine Department, KBMS trainee, Sulaimany teaching hospital, Sulaimany, Kurdistan region, Iraq
}

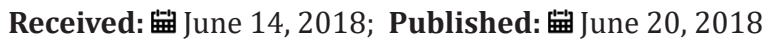

*Corresponding author: Shakhawan M Ali, Maxillofacial Department, KBMS trainee, Surgical Emergency Hospital and Sulaimany teaching hospital, Sulaimany, Kurdistan region, Iraq

\begin{abstract}
Gorlin-Goltz syndrome, is an uncommon, autosomal dominant inherited disorder, which is characterized by numerous basal cell carcinoma (known as nevoid basal cell carcinoma syndrome (NBCCS)). The common manifestations include multiple odontogenic keratocysts in the jaws are usually one of the first manifestations of the syndrome, macrocephaly, frontal boosing, multiple basal cell nevi on the skin with an early age onset, palmar pits, bifid or splayed ribs, high arched palate, calcified diaphragm sellae, calcifications of the falx cerebri. Although all the manifestations may not be present in all the patients. We reported here a child patient, a 8-year-old child boy visited maxillofacial department in Rizgary teaching hospital for right premaxillary swelling emphasizing its clinical and radiographic manifestations of Gorlin -Goltz syndrome with no family history, the patient has multiple odontogenic keratocysts, rib anomalies, calcifications of the falx cerebri, frontal and temporal boosing, macrocephaly and thick eye brows. This study highlights the importance of maxillofacial surgeon in the early diagnosis, treatment and closes follow up is important to prevent long-term complication including malignancy and oromaxillofacial deformation and destruction.
\end{abstract}

Keywords: Gorlin-Goltz; Odontogenic keratocysts; Basal cell; Bifid rib

Abbreviations: NBCCS: Nevoid Basal Cell Carcinoma Syndrome; CT: Computed Tomography; GGS: Gorlin-Goltz syndrome; OPG: Orthopantomogram; BCC: Basal cell carcinoma; MRI: Magnetic Resonance Imaging

\section{Introduction}

Gorlin-Goltz syndrome (GGS) is an uncommon, autosomal dominant inherited disorder known as nevoid basal cell carcinoma syndrome (NBCCS) [1]. This syndrome characterized mainly by the presence of multiple basal cell carcinomas, odontogenic keratocysts of the jaw and palmar and plantar epidermal pits also associated with a wide spectrum of developmental anomalies and neoplasms [2], hypertelorism, calcification of falx cerebri, palmer and planter pits, central nervous system, ocular lesions, rib anomalies, mandibular prognathism (class III jaw relationship), cleft lip and palate Other findings of this syndrome in rare cases ovarian fibromas [3]. Early diagnosis In the case of NBCCS it is of great importance to reduce the severity of complications, such as malignant skin and brain tumors and maxillofacial deformities related to the jaw cysts can be avoided [4]. The dentist has the privilege of diagnosing the

syndrome at first, as mulᄀtiple odontogenic keratocysts are the first manifestations of this syndrome [5]. This article reports a case of a 8-year-old boy patient with GGS, emphasizing its clinical and radiographic manifestations. A case of Gorlin-Goltz syndrome is presented here in which most of the above-mentioned findings are evident.

\section{Case Report}

A 8-year-old child boy patient visited the maxillofacial department in rizgary teaching hospital for right premaxillary swelling for 1 month duration gradually increase in size, now which was associated with pain. Past medical history not significant. Extraoral examination revealed macrocephaly [measuring the head circumference was $58.5 \mathrm{~cm}$ (normal is $49 \mathrm{~cm}$ )], frontal and 
temporal bossing, hypertelorism [Increased intercanthal distance $38 \mathrm{~mm}$ normal is $32 \mathrm{~mm}$ ], wide nasal bridge and thick eye brows (Figure 1). The skin of his palms and soles showing hyperkeratosis and brownish pin point papules in the neck, chest and forearm abnormal chest (Dove chest) (Figures 2 \& 3). Intraoral examination swelling was present on the right premaxillary region revealed bone expansion around unerupted right central and lateral incisor, the overlying mucosa was firm to hard in consistency and was tender on palpation (Figure 1). Radiographic investigations orthopantomogram (OPG) of the patient showed multiple cysts (radiolucencies) present in both maxilla and mandible (Figure 4). CT of the skull revealed calcification of falx cerebri (Figure 5). And a chest radiograph showed bifid ribs (Figure 2). Echocardiography shows mitral valve calcification. Based on clinical history and radiographic findings the case was provisionally diagnosed as Gorlin Goltz syndrome. Surgical enucleation of the cystic lesions was done and the histopathology of the excised epithelium revealed the presence of stratified squamous epithelium with parakeratinization overlying the connective tissue. Prominent palisade basal cell layer with dark-staining nuclei and a corrugated surface with parakeratinization. thereby, confirming the diagnosis of keratocystic odontogenic tumor (Figure 6). Therefore by clinical features, imaging characteristics and histological findings lead to a final diagnosis of Gorlin-Goltz syndrome. We advised the parents of the child about the consequences of his disease, including avoiding sun exposure because of the possibility of BCC development over the face, head and neck, Annual MRI for the brain because of the possibility of brain tumor development (medulloblastoma).

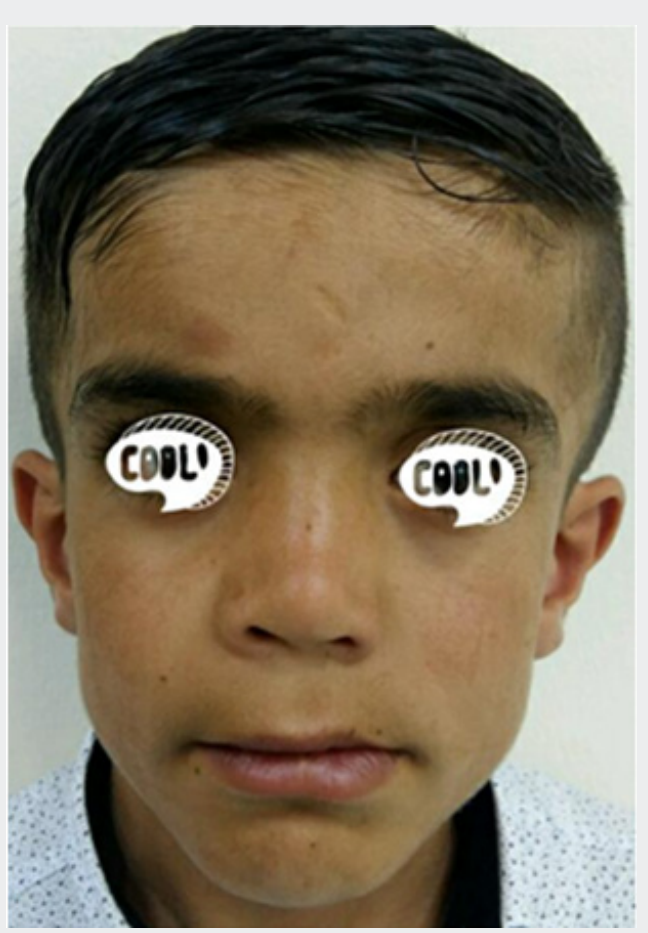

Figure 1: Showing frontal and temporal boosing, hypertelorism, wide nasal bridge, thick eye brows with swelling in the right premaxillary region.

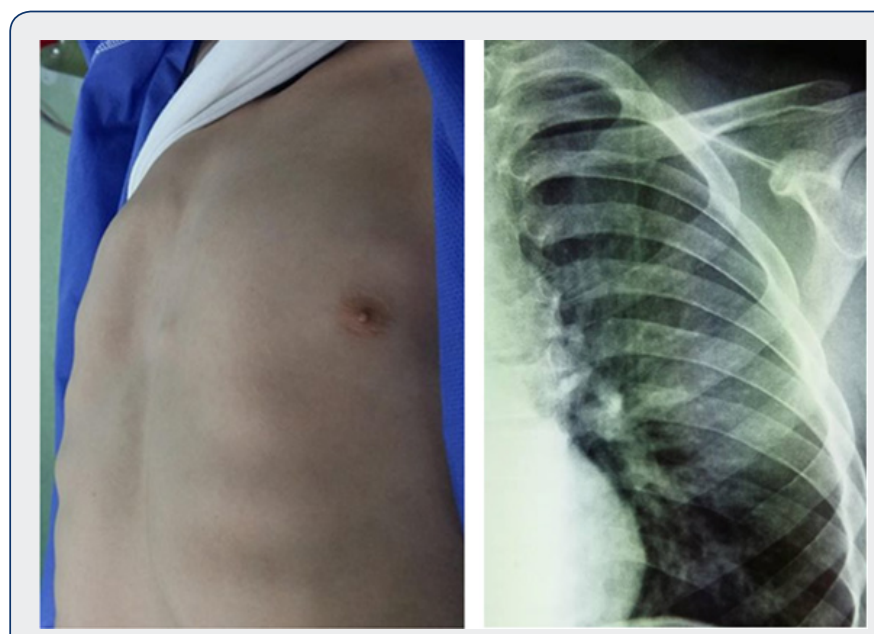

Figure 2: Abnormal chest (Dove chest), PA Chest revealed bifid rib.
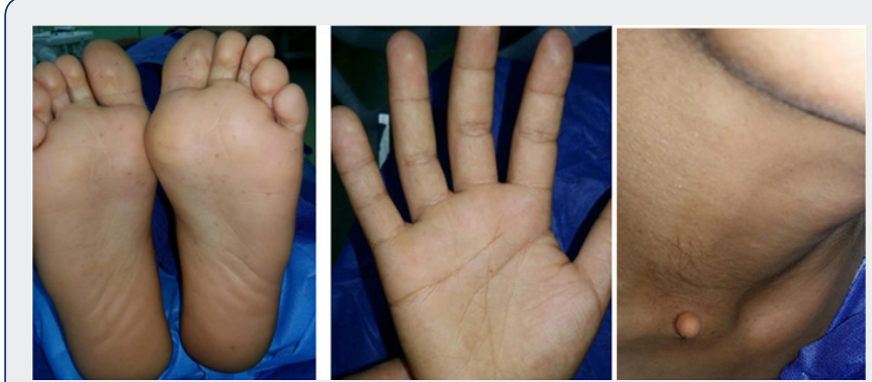

Figure 3: Palmar and plantar epidermal pits with skin tags.

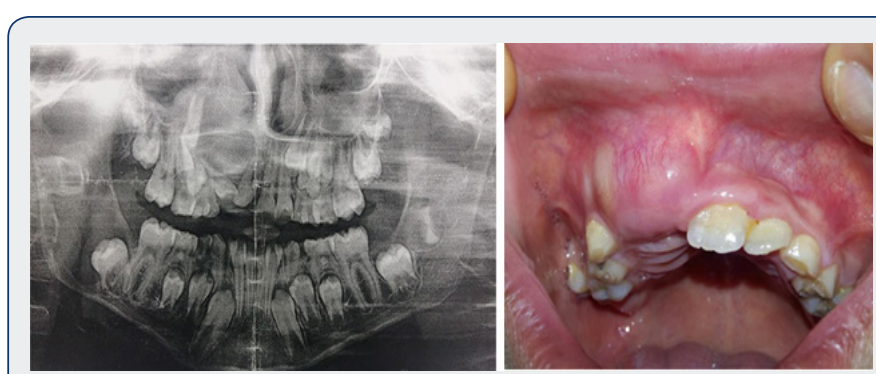

Figure 4: Show multiple OKCs in both maxilla and mandible.

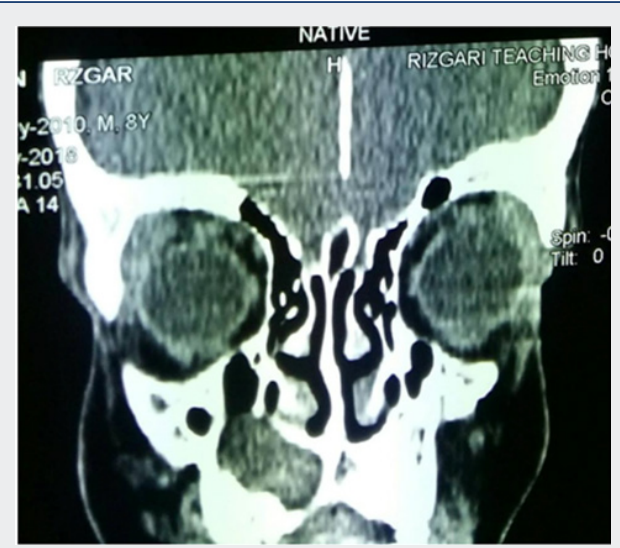

Figure 5: Coronal CT skull showed calcification of falx cerebri. 


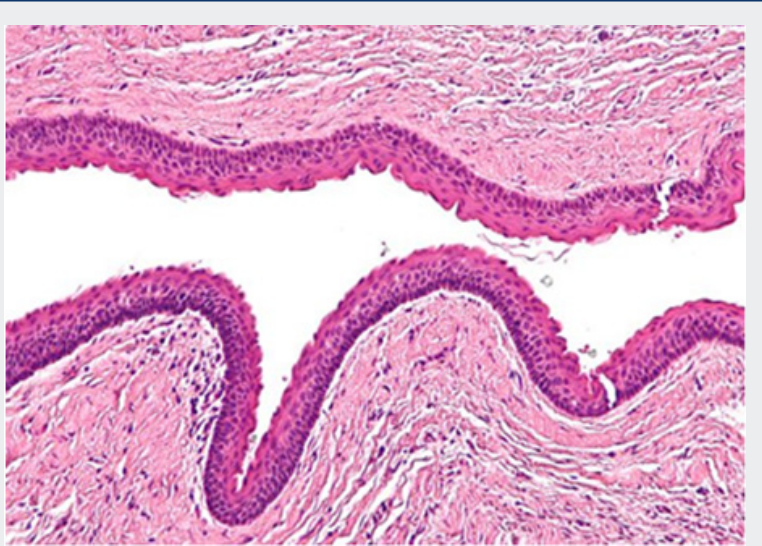

Figure 6: Show prominent palisade basal cell layer with dark-staining nuclei and a corrugated surface with parakeratinization in histopathology findings confirm odontogenic keratocyst.

\section{Discussion}

Gorlin-Goltz syndrome for the first time in 1894 was described by Jarisch and White. The disease is an autosomal dominant disorder mainly characterized by the presence of multiple basal cell carcinomas, odontogenic keratocysts of the jaw and palmar pits [6,7]. It is seen in males and females equally [8]. It is caused by mutations in PTCH, a tumor suppressor gene, a human homologue of a Drosophila segment polarity gene PTCH located in a long arm of chromosome 9q22.3. The prevalence of Gorlin syndrome is estimated to be about 1 in 60,000 [9]. Previously reported GGS presented to the emergency department as facial swelling [10]. In order to be able to establish an early diagnosis of NBCCS, specialists should carry out clinical and imaging examinations in early ages of life [11]. Evans, et al. in 1994 first established major and minor criteria for the diagnosis of this rare entity, which was modified later by Kimonos et al., in 2004 [8,12]. 2 major criteria or 1 major and 2 minor criteria are obligatory in order to diagnose GorlinGoltz syndrome [13].

\section{The Major and Minor Criteria are as Follows}

\section{Major Criteria Consist of}

a) More than two basal cell carcinomas or one in patient less than 20 years old

b) Odontogenic keratocysts of the jaw

c) Bilamellar calcifications of falxcerebri and tentorium

d) Three or more palmar or plantar pits

e) Bifid or fused, or markedly splayed ribs

f) First-degree relative with Gorlin-Goltz syndrome.

\section{Minor Criteria Consist of}

a) Macrocephaly b) Congenital anomalies (cleft lip or palate, frontal bossing, coarse facies, and moderate or severe hypertelorism)

c) Other skeletal anomalies (Sprengel deformity, marked pectus deformity, and marked syndactyly of the digits)

d) Radiologic anomalies (such as bridging of the sellaturcica, vertebral anomalies, modeling defects of the hands and feet, or flame-shaped lucencies of the hands and the feet)

e) Medulloblastoma, seizures, mental retardation, meningioma.

f) Ovarian fibroma

In our case, three major criteria - odontogenic keratocysts of the jaw, calcification of falx cerebri and bifid rib in left side of chest with most minor criteria Macrocephaly, frontal and temporal bossing and moderate hypertelorism Other recognizable features of the syndrome were palmar and plantar epidermal pits with skin tags, solitary, pigmented nevus (mole), areas of hyper-pigmentation in the neck and forearm which confirmed the diagnosis as GorlinGoltz syndrome. The treatment of the Gorlin Goltz syndrome is in accordance with the generally accepted rules for the treatment of basal cell carcinomas and keratocysts in other patients. Radiation should be avoided, as it may trigger off the development of other tumors in the adjacent skin areas.

\section{Conclusion}

This case gives the idea that the maxillofacial surgeon or dentist, especially an oral radiologist, pedodontist and orthodontist, has an important responsibility in early diagnosis It is important to recognize the presence of some major criteria with minor criteria to reach a definitive diagnosis of Gorlin Goltz syndrome also should be made by a multidisciplinary approach team comprising maxillofacial surgeon and medical specialists. Early diagnosis, treatment and close follow up is important to provide a better prognosis for the patient and prevent long term complication including malignancy and oromaxillofacial deformation and destruction.

\section{References}

1. Sabbia T, Bovone S, Camera A, Gambini C, Balbi P (1994) Gorlin-Goltz syndrome with odontogenic keratosis. Report on a patient followed for 10 years. Minerva Stomatol 43(7-8): 359-363.

2. Yordanova I, Gospodinov DK (2007) A familiar case of Gorlin-Goltz syndrome. J of IMAB 13(1): 63-67.

3. Dixit S, Acharya S, Dixit P (2009) Multiple odontogenic keratocysts associated with Gorlin-Goltz syndrome. Kathmandu Univ Med J 7(28): 414-418.

4. Amezaga AOG, Arregui OG, Nuño SZ, Sagredo AA, Urizar JMA (2008) Gorlin-Goltz syndrome: Clinicopathologic aspects. Med Oral Patol Oral Cir Bucal 13(6): 338-343.

5. Kannan KS, Sundharam SB, Manikandan R (2006) Nevoid basal cell carcinoma syndrome. Indian J Dent Res 17(1): 50-53.

6. Kolm I, Puig S, Iranzo P, Malvehy J (2006) Dermoscopy in Gorlin-Goltz Syndrome. Dermatol Surg 32(6): 847-851. 
7. Rózyło Kalinowska I, Rózyło T (2002) Odontogenic keratocyst in GorlinGoltz syndrome. Ann Univ Mariae Curie Sklodowska Med 57(2): 79-85.

8. Kimonis VE, Mehta SG, Digiovanna JJ, Bale SJ, Pastakia B (2004) Radiological features in 82 patients with nevoid basal cell carcinoma (NBCC or Gorlin) syndrome. Genet Med 6(6): 495-502.

9. Neville B, Damm D, Allen C, Bouquot J (2009) Oral and Maxillofacial Pathology. ( $3^{\text {rd }}$ edn); Philadelphia: WB Saunders, p. 984.

10. Aloosi SN, Mahmood KA, Ali ShM, Mahmud PKh, Hasan SO, et al. (2018) A Rare Case of Gorlin-Goltz Syndrome. Presented to the Emergency Department as Facial Swelling. Adv J Emerg Med 2(3): e 40.
11. Casaroto (2011) Early diagnosis of Gorlin-Goltz syndrome: Case report. Head \& Face Medicine 7: 2.

12. Evans D, Ladusans E, Rimmer S, Burnell L, Thakker N, et al. (1993) Complications of the naevoid basal cell carcinoma syndrome: results of a population based study. J Med Genet 30(6): 460-464.

13. Sunder SN, Babburi S, Guduguntla S, Raju P (2013) Gorlin-Goltz syndrome: A rare case report. J NTR Univ Health Sci 2(2): 150-153.

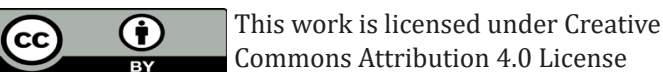

To Submit Your Article Click Here:

Submit Article

DOI: 10.32474/SCSOAJ.2018.01.000115

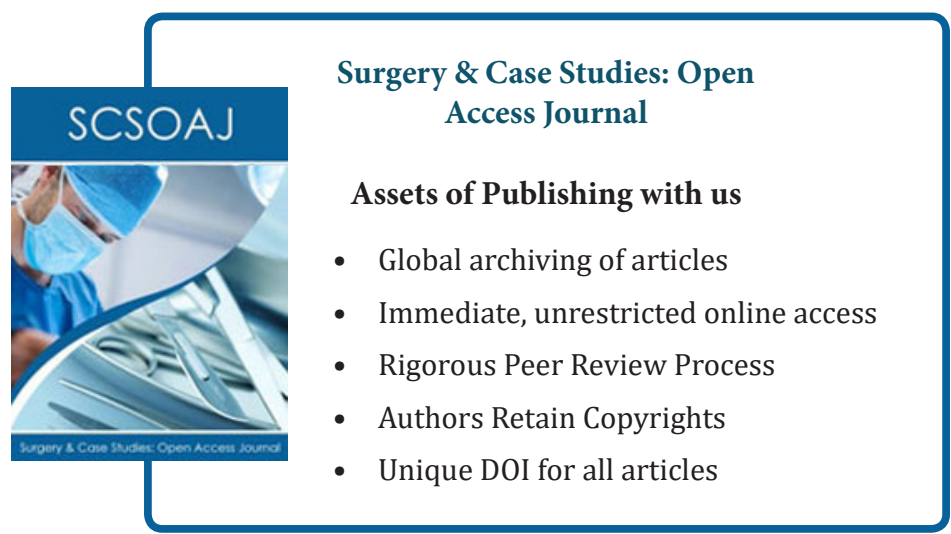

